\title{
Air-Liquid Exchange by Free Hand and One Needle for Unhealed Macular Hole
}

\author{
Haishuang Lin $\left(\mathbb{D},{ }^{1}\right.$ Yiting Ji $\left(\mathbb{D},{ }^{1}\right.$ Luqi Xia $\mathbb{D}^{1},{ }^{1}$ Bo Lin $\left(\mathbb{D},{ }^{2}\right.$ Zhihua Li $\mathbb{D}^{1},{ }^{1}$ Zhixiang Hu $\mathbb{D}^{2}{ }^{2}$ \\ and Ronghan Wu iD $^{2}$ \\ ${ }^{1}$ School of Ophthalmology and Optometry, Wenzhou Medical University, Wenzhou 325027, Zhejiang Province, China
${ }^{2}$ Eye Hospital of Wenzhou Medical University, Wenzhou 325027, Zhejiang Province, China \\ Correspondence should be addressed to Zhixiang Hu; zhixianghucn@163.com and Ronghan Wu; wuronghan@126.com
}

Received 5 October 2021; Accepted 28 December 2021; Published 31 January 2022

Academic Editor: Enrico Peiretti

Copyright (C 2022 Haishuang Lin et al. This is an open access article distributed under the Creative Commons Attribution License, which permits unrestricted use, distribution, and reproduction in any medium, provided the original work is properly cited.

\begin{abstract}
Aim. To report the treatment of 7 cases of unsealed hole after macular hole surgery with air-fluid exchange. Methods. Retrospective case series. We collected 7 eyes of 7 patients with unsealed hole an unsealed hole about 2 weeks after macular hole surgery (23G vitrectomy with internal limiting membrane peeling with sterilizing air tamponade) in our hospital from February 2018 to December 2018. All patients underwent "air-liquid exchange by free hand and one needle." The prone position was taken one week after operation. The macular holes before and after operation were examined by frequency-domain optical coherence tomography (SD-OCT). Results. The size of the macular hole before vitrectomy was $481 \pm 156 \mu \mathrm{m}$ (range: $281-609 \mu \mathrm{m}$ ). Two weeks after vitrectomy (before air and liquid exchange), the size of the macular hole was $295 \pm 92 \mu \mathrm{m}$ (range: $210-421 \mu \mathrm{m}$ ). All macular holes were closed within 7-14 days after air-liquid exchange. There was no complaint of discomfort among these patients. Conclusion. From this preliminary study, air-liquid exchange by free hand and one needle seems to be safe and effective in the treatment for patients with unsealed and tiny macular hole after vitrectomy as the lack of long effective gas in China. However, the exact efficacy and safety need further large case studies.
\end{abstract}

\section{Introduction}

Vitrectomy with internal limiting membrane (ILM) peeling with inflating gas tamponade is the first choice for the treatment of idiopathic macular hole (MH), with a closure rate ranging from $68 \%$ to $98 \%[1,2]$. Some studies show that sterilizing air can also be used for macular hole repair and achieve satisfactory results [3-7]. The advantage of sterilizing air is that the time of prone position is short [8], which is not easy to cause high intraocular pressure. However, the disadvantage is that the absorption time of sterilizing air is short so that the closure rate in the large size of macular hole is lower than inflating gas [9]. Reoperation or gas-liquid exchange with inflating gas is usually used for unsealed hole after macular hole surgery. The operation procedure is complicated which may increase the risk of infection and the burden of patients. Therefore, we report a case series of unsealed hole after macular hole surgery. Patients subsequently underwent "air-liquid exchange by free hand and one needle" with sterilizing air.

\section{Subjects/Materials and Methods}

2.1. Subjects. This case series was retrospective and included 7 eyes from 7 patients with unsealed hole about 2 weeks after macular hole surgery (23G vitrectomy with ILM peeling with sterilizing air tamponade) at the Eye Hospital of Wenzhou Medical University from February 2018 to December 2018.

The inclusion criteria included patients with unsealed hole about 2 weeks after macular hole surgery, and spectral domain optical coherence tomography (OCT) (Spectralis Hra Oct; Heidelberg Engineering, Heidelberg, Germany) showed that the diameter was less than $450 \mu \mathrm{m}$. Exclusion criteria included patients with glaucoma, macular degenerative disease, ocular trauma, ocular tumors, and optic atrophy. Observations regarding macular hole were carried 


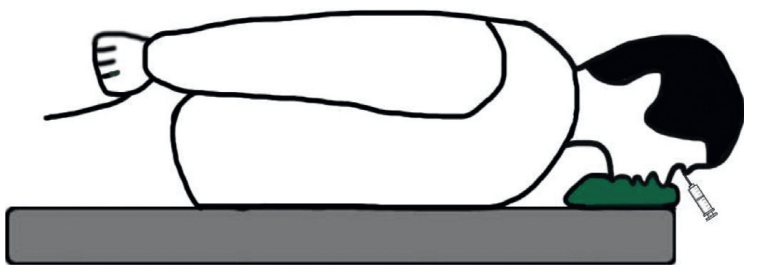

(a)

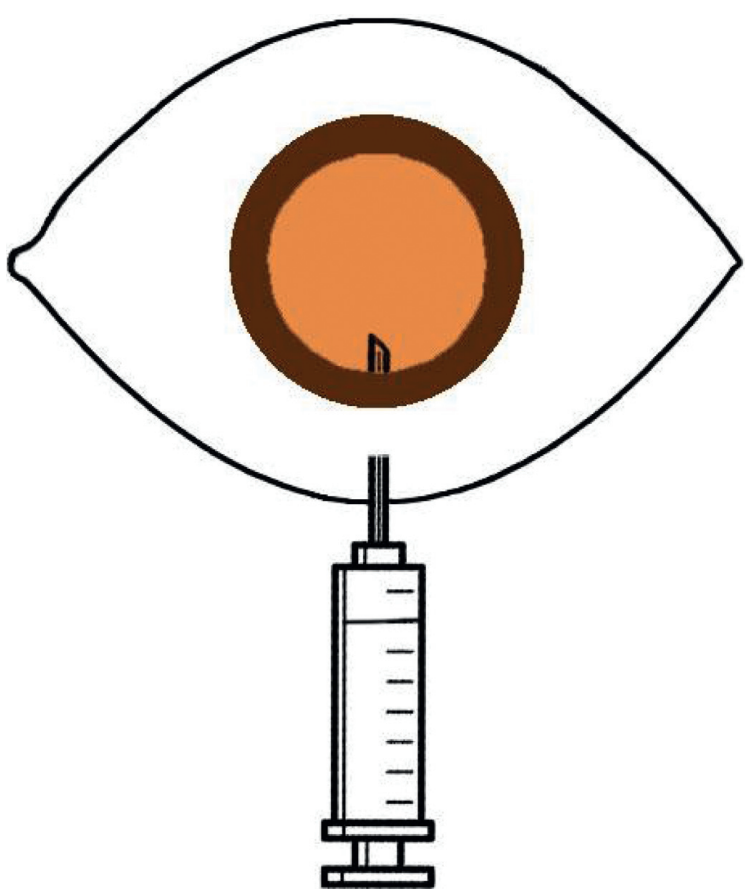

(b)

FIGURE 1: Schematic diagram of "air-liquid exchange by free hand and one needle." (a) Patient took the prone position; (b) the needle was inserted obliquely at $3.5 \mathrm{~mm}$ of the inferior limbus.

out using the SD-OCT. Data obtained included age, gender, affected eye, $\mathrm{MH}$ size before $23 \mathrm{G}$ vitrectomy, $\mathrm{MH}$ size before air-liquid exchange, $\mathrm{MH}$ closure time, and uncorrected visual acuity.

2.2. Surgical Procedure. All patients underwent "air-liquid exchange by free hand and one needle" under topical anesthesia. The patient took the supine position. Anterior chamber penetration was performed to decrease the intraocular pressure into Tn-1 after the conjunctival sac was washed. Then, the patient changed to the prone position. After that, the operating table was raised to the highest position. The patient slightly raised his head and fixed the eye on the supernasal side. Subsequently, $5 \mathrm{ml}$ of sterilizing air was drawn off with a $5 \mathrm{ml}$ syringe, and a $28 \mathrm{G}$ air injection needle was replaced. The needle was placed at the inferior side $3.5 \mathrm{~mm}$ away from the limbus and obliquely inserted into the ocular about $7 \mathrm{~mm}$ (shown in Figure 1(a)). The sterilizing air was injected with vitreous humor was drawn off. Finally, the partial needle was pulled out slowly, and the intraocular pressure was roughly measured by finger sense of the surgeon (shown in Figure 1(b)).

The above-mentioned steps were repeated until the air was observed behind the intraocular lens of the pupil area, and the liquid flow was no longer seen. At this time, the vitreous cavity was filled with air, and the liquid can no longer be drawn off. The liquid drawn off from the vitreous cavity was about $4 \mathrm{ml}$. Subsequently, the needle was pulled out quickly, and the cotton swab was pressed on the puncture port for $3 \mathrm{~min}$. When the needle was pulled out completely, no vitreous humors flowed out. The conjunctival sac was washed again after the patient changed to the supine position. Anterior chamber drainage was performed if intraocular pressure was high. The patients were asked to remain a prone position and to avoid the supine position for one week. All surgeries were performed by one experienced surgeon $(\mathrm{Hu} \mathrm{ZX})$.

\section{Results}

Our "air-liquid exchange by free hand and one needle" technique was performed in 7 patients. The age of 7 patients was $65.4 \pm 6.8$ years, of which three were male. The size of the macular hole before vitrectomy was $481 \pm 156 \mu \mathrm{m}$ (range: 281-609 $\mu \mathrm{m}$ ). Two weeks after vitrectomy (before air and liquid exchange), the size of the macular hole was $295 \pm 92 \mu \mathrm{m}$ (range: $210-421 \mu \mathrm{m}$ ) (shown in Table 1). All macular holes were closed within 7-14 days after air-liquid exchange (shown in Figure 2). The postoperative uncorrected visual acuity had improved from $1.47 \pm 0.59$ logarithm of the minimum angle of resolution (logMAR) units preoreratively to $0.98 \pm 0.30 \log$ MAR units at 1 -month visit after air-liquid exchange $(t=3.131, P=0.02)$ (shown in Table 1). The visual acuity of six patients (85.7\%) improved. There was no complaint of discomfort within these patients in the 1-month follow-up examinations. None of the eyes has required subsequent surgical procedures.

\section{Discussion}

The effective treatment for idiopathic macular hole with smaller diameter (within $600 \mu \mathrm{m}$ ) is vitrectomy with ILM peeling with inflating gas tamponade. The success rate of the 
TABLE 1: Baseline characteristics and postoperative surgical outcome of each patient.

\begin{tabular}{|c|c|c|c|c|c|c|c|c|}
\hline Cases & $\begin{array}{l}\text { Gender/ } \\
\text { age }\end{array}$ & $\begin{array}{l}\text { Operated } \\
\text { eye }\end{array}$ & $\begin{array}{l}\text { Preoperative } \\
\text { visual acuity }\end{array}$ & $\begin{array}{c}\text { Size of } \mathrm{MH} \\
\text { before } \\
\text { vitrectomy }(\mu \mathrm{m})\end{array}$ & $\begin{array}{l}\text { Size of MH two } \\
\text { weeks after } \\
\text { vitrectomy }(\mu \mathrm{m})\end{array}$ & $\begin{array}{c}\text { MH } \\
\text { closure } \\
\text { time (days) }\end{array}$ & $\begin{array}{c}\text { Postoperative } \\
\text { visual acuity at } \\
1 \text { month }\end{array}$ & Complications \\
\hline 1 & $F / 62$ & Right & $\mathrm{FC} / 40 \mathrm{~cm}$ & 609 & 345 & 9 & 0.05 & None \\
\hline 2 & $\mathrm{~F} / 71$ & Right & 0.04 & 567 & 401 & 9 & 0.2 & None \\
\hline 3 & $\mathrm{M} / 53$ & Right & $\mathrm{FC} / 10 \mathrm{~cm}$ & 502 & 421 & 9 & 0.05 & None \\
\hline 4 & $\mathrm{~F} / 64$ & Left & 0.05 & 379 & 223 & 14 & 0.05 & None \\
\hline 5 & $\mathrm{M} / 73$ & Left & 0.08 & 474 & 252 & 7 & 0.2 & None \\
\hline 6 & $\mathrm{M} / 70$ & Left & 0.1 & 555 & 210 & 7 & 0.16 & None \\
\hline 7 & $\mathrm{~F} / 65$ & Right & 0.12 & 281 & 214 & 11 & 0.16 & None \\
\hline
\end{tabular}

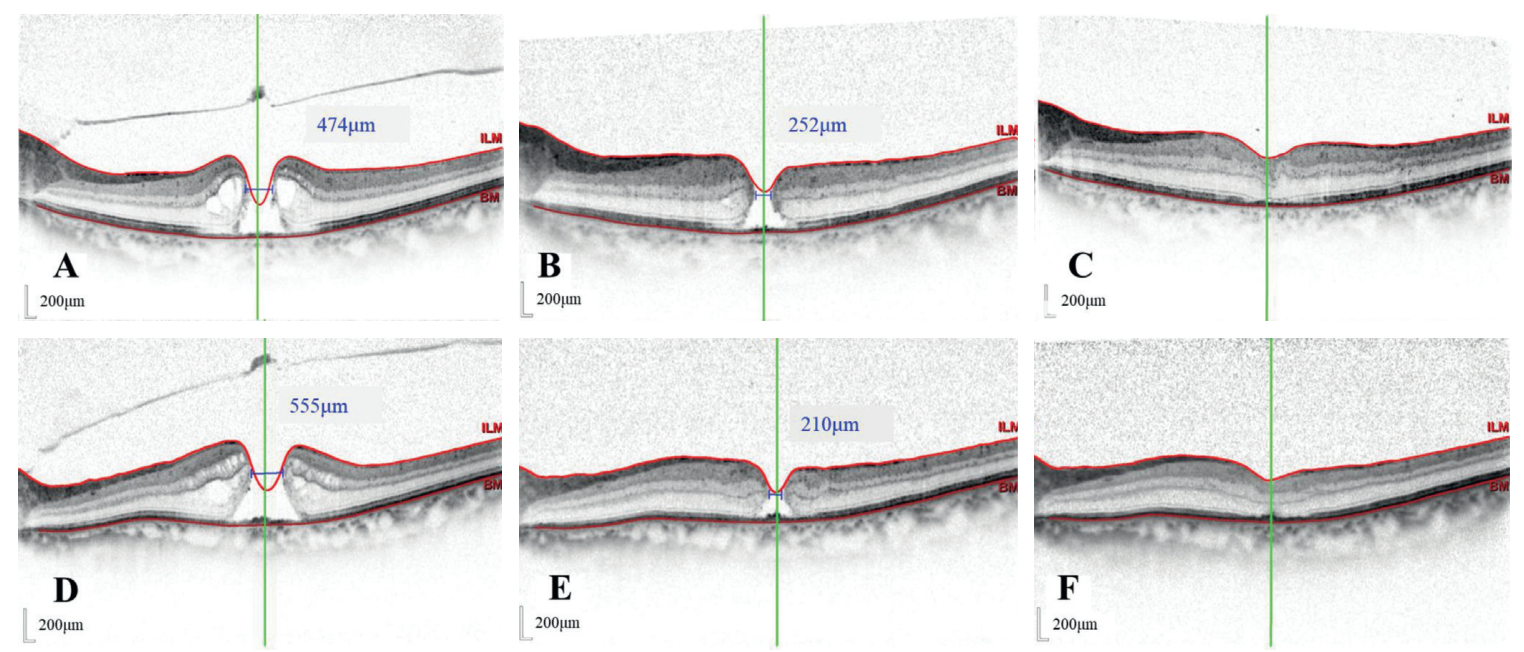

Figure 2: The size of macular holes in three patients before and after surgery. A-C: the size of the macular hole before vitrectomy, before gas-liquid exchange in patient 5, and the macular hole closed 7 days after gas-liquid exchange; D-F: the size of the macular hole before vitrectomy, before gas-liquid exchange in patient 6 , and the macular hole closed 7 days after gas-liquid exchange.

operation is more than $90 \%$ [1]. However, sterilizing air is the main tamponade for the treatment of macular hole, since inflating gas is unavailable in China. Compared with inflating gas, the absorption of sterilizing air is quicker (average 9 days) [10].

There are few studies that compared the effects of sterilized air and inflating gas tamponades on recovery after vitrectomy for the treatment of idiopathic macular hole. He $\mathrm{F}$ et al. pointed out that the primary closure rate was $90.6 \%$ in the sterilized air group and $95.0 \%$ in the perfluoropropane (C3F8) group one month after surgery. There was no statistically significant difference in the closure rate. Also, they concluded that vitrectomy with sterilized air tamponade is safe and effective for the treatment of idiopathic macular hole [9]. Usui $\mathrm{H}$ et al. suggested that air tamponade provided a comparable rate of $\mathrm{MH}$ closure compared with SF6 gas tamponade, at least for $\mathrm{MH}$ with preoperative diameter smaller than $500 \mu \mathrm{m}$ [11]. Forsaa VA et al. proposed that, in the group of full-thickness macular hole $\leq 400 \mu \mathrm{m}$, primary closure occurred in $95 \%$, whereas only $57 \%$ of those $>400 \mu \mathrm{m}$ closed [10].

For patients with unsealed hole after macular hole surgery, revitrectomy with transplantation of residual ILM or other tissue with silicone oil or gas tamponade is usually adopted [12-14]. However, the operation procedure is complicated which may increase the risk of infection. Rao X reported that $89 \%$ patients achieved anatomic success after the outpatient inflating gas-fluid exchange by two needles for open macular hole after primary vitrectomy $[15,16]$. Additional intravitreal inflating gas injection can achieve complete macular hole closure for unsealed macular hole $\leq 700 \mu \mathrm{m}[17,18]$.

In this case series, we treated 7 eyes from 7 patients with an unhealed macular hole within $450 \mu \mathrm{m}$. Complications such as endophthalmitis, high intraocular pressure, vitreous hemorrhage, iatrogenic retinal tear, or retinal detachment have not been observed in the 1-month follow-up. The macular hole of all patients was closed within two weeks after "air-liquid exchange by free hand and one needle." None of the eyes has required subsequent surgical procedures. Compared with two needles, the one needle technique of our case series is easy to operate with controllable intraocular pressure and low risk of infection, which can be completed in the outpatient operating room.

Gas tamponade can restrict the flow of fluid from the vitreous cavity to the macular region, keep the macular region dry, and promote the proliferation and transplantation of glial cells [19]. By additional gas injection, the surface of the macular hole can be kept dry for one week, which can activate the unhealed macular hole to 
reproliferate and repair. The closure of the macular hole can be achieved by additional gas injection even if the macular hole is not closed in the primary macular hole surgery [20-22].

In conclusion, this preliminary study suggests that the new technique "air-liquid exchange by free hand and one needle" using sterilizing air obtained positive results and seems to be safe for eyes with unhealed holes. Long-term anatomic and functional recovery in patients with "air-liquid exchange by free hand and one needle" merit further study.

\section{Data Availability}

The data used to support this study can be obtained from the corresponding author on request.

\section{Ethical Approval}

The study was approved by the Ethics Committee of the Eye Hospital of Wenzhou Medical University (KYK[2018]41). This trial is registered with ChiCTR1800019620. All aspects of the study adhered to the tenets of the Declaration of Helsinki.

\section{Consent}

Each patient was informed about the study, and informed consent was obtained.

\section{Conflicts of Interest}

The authors declare no conflicts of interest.

\section{Acknowledgments}

This work was supported by the Wenzhou Science and Technology Project (Y20210998 to RHW).

\section{References}

[1] B. H. Jr, "Macular hole surgery with and without internal limiting membrane peeling," Archivos de la Sociedad Espanola de Oftalmologia, vol. 107, no. 10, pp. 1939-1948, 2000.

[2] I. P. Chatziralli, P. G. Theodossiadis, and D. H. W. Steel, "Internal limiting membrane peeling in macular hole surgery; why, when, and how?" Retina, vol. 38, no. 5, pp. 870-882, 2018.

[3] J. Lu and Z. Ma, "Clinical outcomes of C3F8 and air tamponade after vitrectomy for the treatment of idiopathic macular hole," Chinese Journal of Ocular Fundus Diseases, vol. 32, no. 5, pp. 473-477, 2016.

[4] Y. Hasegawa, Y. Hata, Y. Mochizuki et al., "Equivalent tamponade by room air as compared with SF6 after macular hole surgery," Graefe's Archive for Clinical and Experimental Ophthalmology, vol. 247, no. 11, pp. 1455-1459, 2009.

[5] L. Hejsek, A. Stepanov, J. Dusova et al., "Microincision 25G pars plana vitrectomy with peeling of the inner limiting membrane and air tamponade in idiopathic macular hole," European Journal of Ophthalmology, vol. 27, no. 1, pp. 93-97, 2017.

[6] F. He, F. Dong, W. Yu, and R. Dai, "Recovery of photoreceptor layer on spectral-domain optical coherence tomography after vitreous surgery combined with air tamponade in chronic idiopathic macular hole," Ophthalmic Surgery, Lasers and Imaging Retina, vol. 46, no. 1, pp. 44-48, 2015.

[7] Y. P. Yu, X. D. Liang, Z. Y. Wang, J. Wang, B. Y. Qi, and W. Liu, "Correlation between diameter and primary closure rate by internal limiting membrane peeling and air tamponade in large idiopathic macular holes," Zhonghua Yan Ke Za Zhi, vol. 55, no. 10, pp. 739-746, 201911.

[8] J. J. Lee, H. J. Kwon, S. M. Lee, I. S. Byon, J. E. Lee, and S. W. Park, "Duration of room air tamponade after vitrectomy," Japanese Journal of Ophthalmology, vol. 64, no. 2, pp. 216-222, 2020.

[9] F. He, L. Zheng, and F. T. Dong, "Comparative study of the effects of sterilized air and perfluoropropane gas tamponades on recovery after idiopathic full-thickness macular hole surgery," Chinese Journal of Ophthalmology, vol. 53, no. 5, pp. 327-331, 2017.

[10] V. A. Forsaa and J. Krohn, "Air tamponade combined with nonsupine positioning in macular hole surgery for pseudophakic eyes," Retina, vol. 37, no. 9, pp. 1750-1756, 2017.

[11] H. Usui, T. Yasukawa, Y. Hirano, H. Morita, M. Yoshida, and Y. Ogura, "Comparative study of the effects of room air and sulfur hexafluoride gas tamponade on functional and morphological recovery after macular hole surgery: a retrospective study," Ophthalmic Research, vol. 50, no. 4, pp. 227-230, 2013.

[12] S. W. Park, K. Y. Pak, K. H. Park, K. H. Kim, I. S. Byon, and J. E. Lee, "Perfluoro-n-octane assisted free internal limiting membrane flap technique for recurrent macular hole," Retina, vol. 35, no. 12, pp. 2652-2656, 2015.

[13] Y. Dai, F. Dong, X. Zhang, and Z. Yang, "Internal limiting membrane transplantation for unclosed and large macular holes," Graefe's archive for clinical and experimental ophthalmology, vol. 254, no. 11, pp. 2095-2099, 2016.

[14] C. Ding, S. Li, and J. Zeng, "Autologous neurosensory retinal transplantation for unclosed and large macular holes," Ophthalmic Research, vol. 61, no. 2, pp. 1-6, 2018.

[15] J. Pieczynski, P. Kuklo, and A. Grzybowski, "Pars plana vitrectomy with silicone oil tamponade for primary and secondary macular hole closure: is it still a useful procedure?" European Journal of Ophthalmology, vol. 28, no. 5, pp. 503514, 2018.

[16] X. Rao, N.-K. Wang, Y.-P. Chen et al., "Outcomes of outpatient fluid-gas exchange for open macular hole after vitrectomy," American Journal of Ophthalmology, vol. 156, no. 2, pp. 326-333, 2013.

[17] M. Imai, T. Gotoh, and H. Iijima, "Additional intravitreal gas injection in the early postoperative period for an unclosed macular hole treated with internal limiting membrane peeling," Retina, vol. 25, no. 2, pp. 158-161, 2005.

[18] T. Iwase and K. Sugiyama, "Additional gas injection after failed macular hole surgery with internal limiting membrane peeling," Clinical and Experimental Ophthalmology, vol. 35, no. 3, pp. 214-219, 2007.

[19] R. Rahman, I. Madgula, and K. Khan, "Outcomes of sulfur hexafluoride (SF6) versus perfluoroethane (C2F6) gas tamponade for non-posturing macular-hole surgery," British Journal of Ophthalmology, vol. 96, no. 2, pp. 185-188, 2012.

[20] C. Gesser, T. Eckert, U. Eckardt, U. Porkert, and C. Eckardt, "Makulalochchirurgie mit Lufttamponade," Ophthalmologe, Der, vol. 107, no. 11, pp. 1043-1050, 2010.

[21] C. Eckardt, T. Eckert, U. Eckardt, U. Porkert, and C. Gesser, "Macular hole surgery with air tamponade and optical 
coherence tomography-based duration of face-down positioning," Retina, vol. 28, no. 8, pp. 1087-1096, 2008.

[22] J. P. Ehlers, A. Uchida, S. K. Srivastava, and M. Hu, "Predictive model for macular hole closure speed: insights from intraoperative optical coherence tomography," Translational Vision Science \& Technology, vol. 8, no. 1, 18 pages, 20196. 